\title{
Prototyping Ubiquitous Imaging Surfaces
}

\author{
Kyle Montague', Daniel Jackson'1, Tobias Brühwiler², Tom Bartindale', Gerard Wilkinson ${ }^{1}$, \\ Patrick Olivier ${ }^{1}$, Otmar Hilliges ${ }^{2}$, Thomas Ploetz ${ }^{3}$ \\ ${ }^{1}$ Newcastle University, \\ Newcastle upon Tyne, \\ United Kingdom
}

\{firstname.surname\}@ncl.ac.uk, g.wilkinson@ncl.ac.uk

\begin{abstract}
Mass adoption and innovation in the field of the Internet of Things has transformed the environments we live in, from stale siloes of technologies into rich interactive playgrounds. Nevertheless, the vast majority of surface area in these spaces are being overlooked and under-utilized in today's research. Surface imaging provides the means to extend and include typically out-of-reach, disconnected objects into these playgrounds. However, existing surface imaging technologies are impractical to embed in everyday environments, restricting researchers from exploring the design and interaction opportunities they can afforded these spaces. In this paper, we propose IRIS, a modular surface imaging prototype capable of providing scalable, low-cost, high-resolution surface imaging. We describe a real-world case study where IRIS is used to identify and track fresh fruit produce being prepared - a task that is typical infeasible with existing technologies. Through IRIS, we hope to enable the community to exploit these under-explored surface areas and enhance the rich, interactive, connected environments we inhabit.
\end{abstract}

\section{Author Keywords}

Surface Imaging; Modular Sensing; Internet of Things.

\section{ACM Classification Keywords}

H.5.2 User Interfaces: Input devices and strategies.

\section{INTRODUCTION}

It was predicted, that in the future all of the spaces in which we work, live and play will be rich with interaction and ubiquitous sensing woven into the environment [17]. Today, we are already experiencing digital sensing and interaction embedded into many aspects of our daily lives. We have connected smart homes that monitor and learn our daily routines with seemingly autonomous control over an entire arsenal of IoT (Internet of Things) devices. With each new

This work is licensed under a Creative Commons Attribution International 4.0 License.

DIS 2017, June 10-14, 2017, Edinburgh, United Kingdom ACM 978-1-4503-4922-2/17/06.

http://dx.doi.org/10.1145/3064663.3064688
Thing added to the network the sensing capabilities and interaction opportunities grow exponentially. Despite the tremendous efforts to make everything part of IoT, there are a wealth of objects that remain disconnected - and therefore invisible to our connected worlds. For objects where it is impractical or infeasible to embed the required digital identification and communication means, we need alternative strategies to include them in our networks and IoT environments.

We believe surface-based imaging in conjunction with Computer Vision hold the key to expanding what is possible within these interactive playgrounds. Nevertheless, we are currently limited by existing imaging technologies, which fail to support the pixel density, form factors and scalability required to achieve truly ubiquitous imaging surfaces. It is undeniable that new interactions can be made possible in spaces where every surface is capable of imaging objects placed onto it; where the physical form of objects can be linked with their provenance and history through time and space. However, it is unclear what this new paradigm of interaction will look like without first embedding the technology - which can be infeasible due to the aforementioned constraints. To investigate the design space and interaction possibilities of ubiquitous imaging surfaces, we developed a modular imaging surface prototype, made with off-the-shelf components. Our modular design enables multiple units to be connected together, supporting the creation and reconfiguration of arbitrary-shaped imaging surfaces, to accommodate a diverse range of surface imaging scenarios.

In this paper, we present IRIS, a practical implementation of ubiquitous imaging surfaces - using cheap, readily-available components. Our hardware designs and software are Open Source and have been made available as a platform to support other designers and researchers exploring ubiquitous imaging environments. Finally, through a case study, we explore the potential application of IRIS for food identification without RFID markers.

\section{RELATED WORK}

The identification and tracking of real-world objects in a space can be valuable in many contexts. Office $[13,14]$, home and social [2] environments all present rich interaction spaces for ubiquitous surface imaging. Historically, single [5,11] and multiple [1] high-resolution cameras 
mounted behind surfaces have been demonstrated as valid approaches to surface imaging, and LCD based technologies such as PixelSense [9], FiberBoard [3] and cameras placed on surface edges [7] have demonstrated how flat form factors can be utilized for optical imaging in low pixel density imaging tasks. However, these solutions are single units which constrains the capture resolution and physical size, and ultimately limits the shape that an imaging surface can be, typically conforming to rectangular or circular forms. In addition, installation often requires significant depth behind or underneath individual units.

Using smaller, individual units combined together is a viable alternative [15], but this work has been limited to large collaborative interaction and display spaces, rather than ubiquitous environmental sensing. As existing surfaces include integrated displays, these technologies typically rely on infrared vision, where the limited spectrum would restrict their ability to differentiate real-world objects.

Computer Vision based tracking has traditionally been performed using video of the environment [8], however surface occlusion becomes a significant issue when identifying objects placed on surfaces being used by people. Some objects can often be 'tagged' in advance using RFID [12] or visual marker [4,6] technologies; however, RF methods do not track orientation, and both techniques fail with objects that cannot be tagged. Moreover, it is not always desirable or possible to embed cameras in an environment, as they capture more than the intended interactions and impose on individuals' privacy. While, surface imaging approaches can rely on embedded cameras, it is possible to maintain privacy by limiting focus and diffusing light to obfuscate anything beyond the imaging surface.

\section{UBIQUITOUS IMAGING SURFACES}

Connected environments need to be aware of objects out of reach of current IoT infrastructure. Surface imaging has the potential to support this need. However, due to technical constraints the design space of imaging surfaces has been limited to traditional form factors. To explore the interaction opportunities and design challenges of truly ubiquitous imaging surfaces, we need functional prototypes that can be embedded into real world environments, with diverse spatial, contextual and privacy demands.

To ensure our approach could support a wide range of imaging surface scenarios, we adopted the following conceptual principles:

\section{Configurable and Malleable}

The world we live in is not comprised of solely rectangular surfaces - nor is it likely that surface imaging will impose these rules in the future. Therefore, we must be able to easily create arbitrary-shaped imaging surfaces that conform to the environment.

\section{Scalable}

Our environments consist of surfaces large and small. The dimensions of a surface can restrict or promote behaviors and interactions. Ubiquitous imaging surfaces must be suitable for the same dimensional configurations as used in our existing environments.

\section{Spatial Reconfiguration and Extensibility}

The transient nature of surfaces within environments can lead to changes in their spatial configuration. For example, as one table is moved adjacent to another, the physical interactions often extend to occupy the new space. Ubiquitous imaging surfaces should respond appropriately, e.g. by exchanging local surface knowledge with each other. These surfaces are prime locations for interaction and collaboration, therefore it is vital that ubiquitous imaging surfaces can respond to this demand of spatial reconfiguration and extensibility.

\section{Self-Organizing}

Furthermore, the spatial configuration of objects, such as grouping or clustering, can hold an intrinsic meaning to interactions. As such, at the unit level, the spatial configuration of imaging of surfaces must be known.

\section{Acceptability}

A fundamental barrier to the adoption of technology is its acceptability within society. The appearance and privacy norms of environments should be reflected in the form and function of ubiquitous imaging surfaces.

\section{IRIS DESIGN}

We approach the challenge of creating a ubiquitous imaging surface by moving away from the notion of a single imaging device per surface, to a collection of smaller devices working cooperatively. U-Texture demonstrated a similar approach, using inter-connecting touchscreen displays, to created smart objects [16]. Distributing a surface between multiple cameras allows a high pixel density while minimizing the overall depth required, and yet the scale is still limited in two important ways. Firstly, at some magnitude, camera bandwidth and image computation will exceed a single processing unit's capabilities - indicating a need to distribute the processing. Still, at some scale, distributed processing using a single physical medium for communication between

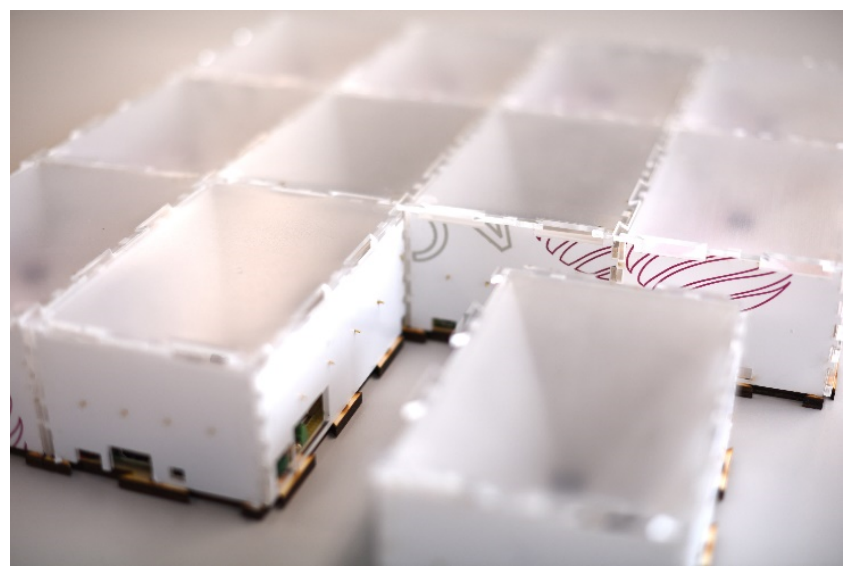

Figure 1. The modular design of an IRIS surface showing the interlocking, tessellating units. 
nodes (e.g. a wireless network or un-switched Ethernet connection) would saturate that channel - indicating that a non-global network topology must be used.

In response to these challenges, IRIS uses a modular design, which allows larger surfaces to be composed from multiple smaller IRIS units, each with a single camera and processor for local surface imaging. This decentralized approach where each module has its own processing ability and direct, local communication with neighboring modules - frees the modular construction from shape and scale limitations.

\section{Hardware Components}

Each IRIS unit (Figure 1 and 2) is an identical, self-contained imaging surface. A unit is compact $(160 \times 105 \times 73 \mathrm{~mm})$ and designed to be connected to form a larger surface, supported by power and data coupling along each edge. A camera (Raspberry Pi Camera 2) with a wide field of view (5MP $160^{\circ} \mathrm{FOV}$ ) is used to obtain a high pixel density image of the local surface $(1944 \times 1458$ pixel color, after correcting for distortion yields $170-300 \mathrm{dpi}$ ). The image is processed locally by the embedded computer (Raspberry Pi 3 Model B). A four-channel UART (FT4232H mini module) provides bi-directional serial communication with adjacent boards via sprung pogo pins and contact pads mounted on the sides of the unit, which also distribute power. Optional LED rear-illumination to improve color and contrast is switched through a Darlington array (ULN2803). Finally, a battery allows the devices to continue to continue to operate and maintain state during brief periods of disconnection (e.g. rearrangement of devices).

The unit enclosure is an inter-locking, tessellating design made from laser-cut acrylic sheets with an acrylic top surface and plywood base. This configuration allows the units to quickly be snapped together or extended to create larger surfaces, as illustrated in Figure 3. Power transfer and local communication is achieved via the physical contact made by units when connected together. IRIS units determine their local topology and are hot-swappable, allowing imaging surfaces to be reconfigured and individual units to be replaced as required.

The top surface can be diffused so that objects away from the surface are blurred. This configuration offers better privacy for people otherwise within the camera's field of view, without impairing the high-resolution imaging of objects placed directly on the unit. An additional benefit is that the diffusion allows for more robust image segmentation. Furthermore, we ensure that objects remain visible when the unit has little or no ambient light by using a backlight diffuser with white LEDs to illuminate the subjects from below.

\section{Image Processing}

IRIS units capitalize on the available hardware acceleration within the processor by directly delivering the camera image to the GPU (via a CSI bus). The units use a wide-angle camera lens to reduce the depth of the device, yet this produces an image that suffers from strong barrel distortion.

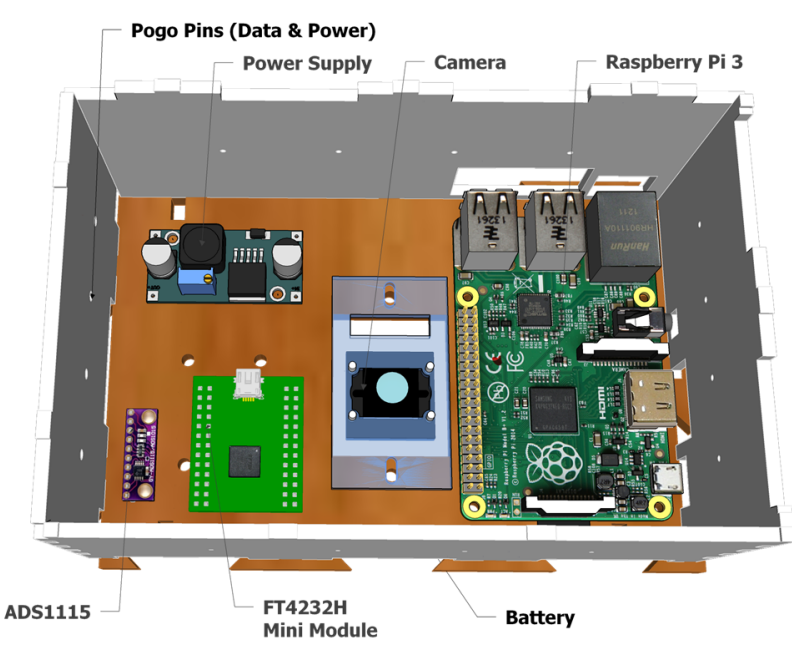

Figure 2. The internal layout of a single IRIS unit with components labelled.

We create an un-distortion map, tailored to each device with a semi-automated calibration procedure, loaded into the GPU to allow efficient correction of the incoming images.

The system uses two schemes of object classification: recognition of object type, and identification of unique objects. Using the un-distortion map, we extract a lowresolution $(256 \times 192$ pixel $)$ image and segment the foreground from the background by thresholding the saturation channel to create an image mask. From the resulting image, we can perform object recognition by processing the image for contours. We then match these contour features to recognize the type of object. With the bounds of the resulting object candidate contours, we extract high-resolution (up to $1944 \times 1458$ resolution) patches from the original image. Each patch is processed for Speeded-Up Robust Features (SURF) to perform object identification using a brute force matcher.

\section{Communication between units}

As objects can be freely placed on the IRIS surface, and particularly given the small surface area of a single unit, objects can be expected to be positioned across multiple

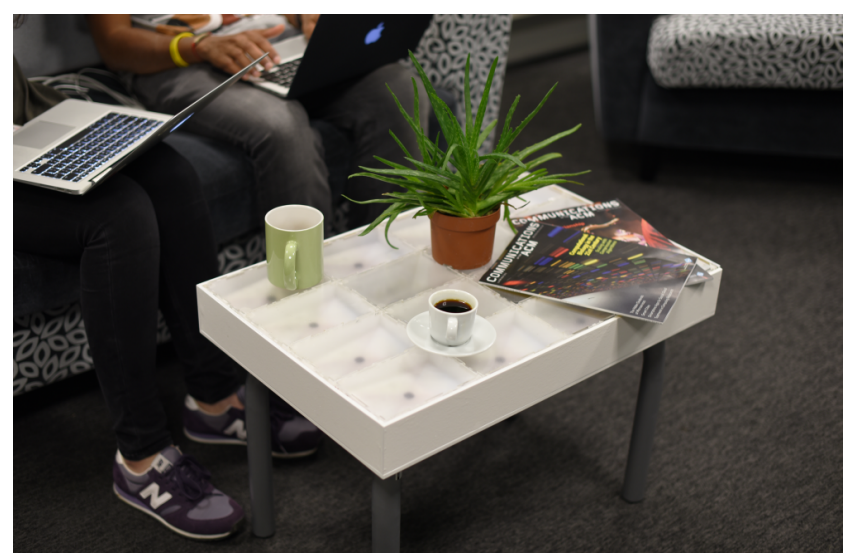

Figure 3. A coffee table created from 16 IRIS units. 


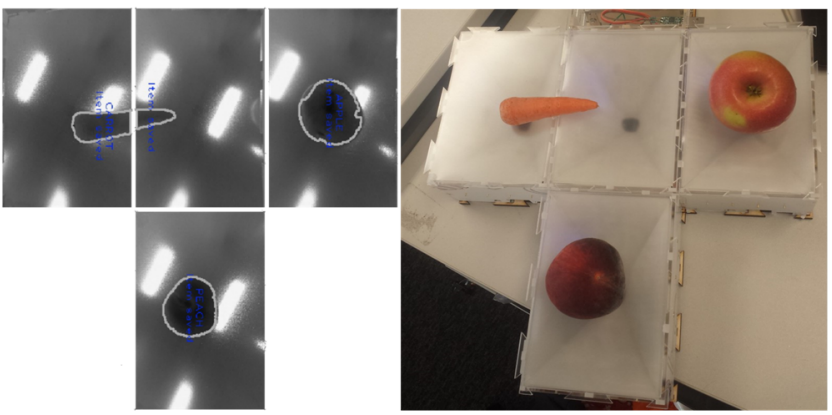

Figure 4. IRIS surface identifying fresh food. Left: the composite image as seen and labelled by the surface, right: the scene viewed from above.

units. IRIS supports determining object identification between multiple units by exchanging extracted features and object definitions with connected neighbors. When a contour patches lies along the border of two units, their features (object contour and SURF extracted points) are automatically communicated to the adjacent unit; this reciprocal action allows both devices to independently process the features for object candidates.

Networking is achieved via a physical connection to the immediately attached neighboring units. When the devices are snapped together, four pogo pins and pads make contact from adjoining edges. Two connections provide common power rails (positive voltage and ground), and two provide bidirectional serial communication (transmit and receive).

A collective catalogue of objects known to the surface is maintained through each unit recursively sharing object definitions and features with their neighbors. This approach is beneficial because it allows any unit to extend the object catalogue; additionally, as new units are introduced to the surface to extend it, they too are notified of the existing object catalogue and can immediately begin contributing to the imaging tasks.

\section{CASE STUDY: FOOD IDENTIFICATION}

Radio Frequency identification (RFID) tags revolutionized large-scale commercial product identification and tracking by enabling individual goods to be assigned unique identities; thus, supporting persistent and historical tracking of their life-cycle. While a large proportion of consumer goods are already being shipped with some form of identification marker, it is not always physically possible or sustainably viable to embed electronic tags in goods such as fresh foods. For these items, we are currently unable to take advantage of the interaction opportunities such identification can afford, rendering these goods invisible to today's IoT and connected environments. Work has proved, however, that surface image recognition of these items can be successful given appropriate image information [10].

We conducted a case study to explore the interaction opportunities of uniquely identifying these objects. Using our IRIS prototype platform, we built an RFID alternative for identifying and tracking fresh foods. IRIS is capable of distinguishing between multiple types of fresh foods (including apples, carrots, oranges, onions and peaches), moreover IRIS can uniquely identify specific instances of fresh food. Each piece of fresh food was assigned its own unique identification number; by placing it on to the IRIS surface. Once added to the surface's catalogue of objects, the fresh food could be successfully moved around and identified anywhere across the surface.

In initial testing, object feature descriptors included a total of 38 active variables extracted from the SURF algorithm, this configuration produced a recognition accuracy of $97.2 \%$ (five categories, three instances of each category). While this case study used a relatively small catalogue of objects, the results were promising and demonstrate the potential for this type of ubiquitous imaging application, shown in Figure 4.

\section{CONCLUSION AND FUTURE WORK}

In this paper, we have discussed the technical challenges and design opportunities of ubiquitous imaging surfaces to enhance our connected environments. Recognizing the need for functional technologies to enable designers and researchers alike to explore this exciting interactive space, we created IRIS, an Open Source imaging surface prototype. IRIS is a modular high pixel density surface that can be scaled and physically reconfigured to create arbitrary shaped planar imaging surfaces. IRIS contributes a practical approach towards extensible, ubiquitous imaging surfaces using off-the-shelf components.

We acknowledge our solution is a stepping stone to true ubiquitous imaging surfaces, and as camera, battery and networking technologies improve, so will the form-factor and availability of this type of sensor. We envision a world where imaging surfaces are like stickers, cameras are consumables and can be placed anywhere, without preparation, by anyone. Using currently available technology, we have demonstrated the potential for IRIS to extend the IoT, and connected environments to include previously excluded everyday objects through high resolution imaging to support object identification.

Our contribution stands as a practical and cost-effective solution for creating scalable imaging surfaces. We encourage the community to build upon this platform to explore the design space and create increasingly connected environments using ubiquitous surface imaging.

\section{ACKNOWLEDGEMENTS}

This project was funded by EPSRC award number DERC EP/M023001/1 (Digital Economy Research Centre). All source code and hardware designs are available at: https://github.com/digitalinteraction/iris

\section{REFERENCES}

1. YoungSeok Ahn, HyungSeok Kim, Mingue Lim, Jun Lee, and Jee-In Kim. 2010. A slim tabletop interface based on high resolution LCD screens with multiple 
cameras. In ACM International Conference on Interactive Tabletops and Surfaces - ITS '10, 241. http://doi.org/10.1145/1936652.1936696

2. Till Ballendat, Nicolai Marquardt, and Saul Greenberg. 2010. Proxemic interaction. In ACM International Conference on Interactive Tabletops and Surfaces ITS '10, 121. http://doi.org/10.1145/1936652.1936676

3. Daniel Jackson, Tom Bartindale, and Patrick Olivier. 2009. FiberBoard: compact multi-touch display using channeled light. In ACM International Conference on Interactive Tabletops and Surfaces - ITS '09. 25-28. http://doi.org/10.1145/1731903.1731908

4. Mark Fiala. 2005. ARTag, a fiducial marker system using digital techniques. In IEEE Computer Society Conference on Computer Vision and Pattern Recognition 2005 (CVPR '05), vol. 2, 590-596. http://doi.org/10.1109/CVPR.2005.74

5. Christian Holz and Patrick Baudisch. 2013. Fiberio. In Proceedings of the 26th annual ACM symposium on User interface software and technology - UIST '13, 4150. http://doi.org/10.1145/2501988.2502021

6. Martin Kaltenbrunner and Ross Bencina. 2007. reacTIVision: a computer-vision framework for tablebased tangible interaction. In TEI, 69-74. Retrieved from http://portal.acm.org/citation.cfm?id=1226969.1226983

7. Otto Korkalo and Petri Honkamaa. 2010. Construction and evaluation of multi-touch screens using multiple cameras located on the side of the display. In $A C M$ International Conference on Interactive Tabletops and Surfaces - ITS '10, 83. http://doi.org/10.1145/1936652.1936667

8. Girish Kulkarni, Visruth Premraj, Sagnik Dhar, et al. 2011. Baby talk: Understanding and generating simple image descriptions. In CVPR 2011, 1601-1608. http://doi.org/10.1109/CVPR.2011.5995466

9. Microsoft. 2012. The Power of PixelSense ${ }^{\mathrm{TM}}$. Retrieved November 12, 2012 from http://www.microsoft.com/enus/pixelsense/pixelsense.aspx
10. Cuong Pham, Daniel Jackson, Johannes Schoening, Tom Bartindale, Thomas Ploetz, and Patrick Olivier. 2013. FoodBoard. In Proceedings of the 2013 ACM international joint conference on Pervasive and ubiquitous computing - UbiComp '13, 749. http://doi.org/10.1145/2493432.2493522

11. Johannes Schöning, Jonathan Hook, Nima Motamedi, et al. 2009. Building Interactive Multi-Touch Surfaces. Journal of Graphics, GPU, and Game Tools 14, 3: 3555. http://doi.org/10.1080/2151237X.2009.10129285

12. Roy Want, Kenneth P. Fishkin, Anuj Gujar, and Beverly L. Harrison. 1999. Bridging physical and virtual worlds with electronic tags. In Proceedings of the SIGCHI conference on Human factors in computing systems the CHI is the limit - CHI '99, 370-377. http://doi.org/10.1145/302979.303111

13. Pierre Wellner and Pierre. 1991. The DigitalDesk calculator. In Proceedings of the 4th annual ACM symposium on User interface software and technology UIST '91, 27-33. http://doi.org/10.1145/120782.120785

14. Daniel Wigdor, Gerald Penn, Kathy Ryall, Alan Esenther, and Chia Shen. 2007. Living with a Tabletop: Analysis and Observations of Long Term Office Use of a Multi-Touch Table. In Second Annual IEEE International Workshop on Horizontal Interactive Human-Computer Systems (TABLETOP'07), 60-67. http://doi.org/10.1109/TABLETOP.2007.33

15. Multitaction. 2016. Multitaction - Collaboration Made Easy. Retrieved September 21, 2016 from https:/www.multitaction.com/

16. Naohiko Kohtake, Ryo Ohsawa, Takuro Yonezawa, et al. 2005. u-Texture: Self-Organizable Universal Panels for Creating Smart Surroundings. In UbiComp 2005: Ubiquitous Computing. Springer, Berlin, Heidelberg, 19-36. http://doi.org/10.1007/11551201_2

17. M Weiser. 1995. The computer for the 21st century. Scientific American. 265(3):94-104. 